\title{
DENOMINACIONES GEOGRÁFICAS PROTEGIDAS (DGP): OPORTUNIDAD COMERCIAL PARA EL PATRIMONIO CENTROAMERICANO
}

\author{
ALONSO RODRÍGUEZ CHAVES \\ ESCUELA DE CIENCIAS SOCIALES \\ Universidad Estatal a Distancia \\ arodriguez@uned.ac.cr
}

\section{RESUMEN}

El artículo se basa en referentes que destacan la importancia de las Denominaciones Geográficas Protegidas (DGP) como novedosos instrumentos comerciales a desarrollar en la región centroamericana. Por ello se invita al lector a conocer los elementos que limitan e igual favorecen la implementación de dicho sistema en la región. Principalmente, se considera en el estudio la abundante riqueza patrimonial y diversidad de productos que gozan de gran prestigio a nivel internacional y que en el transcurso del tiempo, han venido a representar símbolos de identidad regional.

PALABRAS CLAVES: DENOMINACIONES GEOGRÁFICAS, DISTINCIONES GEOGRÁFICAS, PATRIMONIO CULTURAL TANGIBLE E INTANGIBLE, VENTAJAS COMPETITIVAS, CONSUMIDORES GOURNET, PRODUCTOS NOSTÁLGICOS.

\section{ABSTRACT}

The article is based on references that highlight the importance of Protected Geographical Names (DGP) as innovative tools to develop business in Central America. Therefore invites the reader to understand the elements that limit and equal favor the implementation of this system in the region. Mainly, it is considered in the study, the abundant wealth and diversity of products that are highly respected internationally and over time have come to represent symbols of regional identity.

KEYWORDS: GEOGRAPHICAL DENOMINATIONS, GEOGRAPHICAL DISTINCTIONS, TANGIBLE AND INTANGIBLE CULTURAL HERITAGE,
COMPETITIVE ADVANTAGE, CONSUMERS GOURNET, NOSTALGIC PRODUCTS.

\section{INTRODUCCIÓN}

El trabajo trata de las posibilidades de implementar las Denominaciones Geográficas en el ámbito centroamericano. Con esto no solo se limita a comprender la importancia de los instrumentos sino la potencialidad y viabilidad que tienen sus productos para optar a tales distinciones.

En ese sentido, propone romper con esquemas tradicionales e incluye la idea de extender las denominaciones geográficas a otros productos, bienes y servicios originarios, dado su interés económico, la tradición, así como sus cualidades situadas por encima de otros productos similares.

Bajo esta línea se enfatiza en conocer planteamientos y acciones institucionales y de productores a escala regional y nacional, que aproximen a su implementación. Ante ello, el eje de estudio contempla el marco de discusiones internacionales, convenios y tratados bi y multinacionales. En particular estándares mínimos dispuestos por la Unión Europea, la Organización Mundial del Comercio y otros.

Tras esta estructura, vale aclarar las estrategias metodológicas utilizadas. Para empezar nos referimos a que la misma se circunscribe dentro del marco investigativo con enfoque cualitativo. Esta reflexión es trascendental, pues el objeto de estudio a examinar se manifiesta en esa vertiente. 
Con esa lógica, la investigación se fundamentó más en un proceso inductivo, en el que se exploró, describió y luego se procedió a generar perspectivas teóricas, que giran de lo particular a lo general. En términos generales, se partió de la idea que existen estudios descriptivos previos que han detectado y definido ciertas variables y generalizaciones, pues descubrirlas permite a su vez fundamentarlo.

Al constituir apenas una incipiente aspiración y al existir un relativo desconocimiento sobre el tema, la investigación se perfila de manera inicial un tanto exploratoria. Pues el objeto de estudio es relativamente novedoso y desconocido en su aplicación. En este caso se recopiló información que permitiera diagnosticar y fundamentar a manera de propuesta, la posibilidad de llevar a cabo tanto la aplicación como el desarrollo de las denominaciones geográficas en el contexto particular.

Las fuentes anteriores, fueron complementadas con los escasos estudios e intervenciones de especialistas, que ayudaron a defender posturas posibilistas, tanto que emergen alrededor en la región centroamericana como cercanas a ella. Igualmente, para atender el "vínculo" específico entre los productos y el lugar de origen, se visitó algunas comunidades donde se perciben incipientes organizaciones de productores interesados en desarrollar prácticas relacionadas con las denominaciones geográficas protegidas por sus características históricas-geográficas.

En consecuencia se propuso el uso de unidades de análisis recomendadas por Lofland y Lofland, quienes sugieren en la naturaleza de un proceso como el presentado, desarrollar encuentros presenciales, cuyo objetivo era complementar e intercambiar información con productores rurales que han desarrollado prácticas innovadoras y que han obtenido resultados satisfactorios en el comercio nacional e internacional.

La metodología utilizada se cifra en la aplicación de técnicas cualitativas de investigación so- ciocultural, tanto para la recolección como para el análisis de los datos recopilados y sistematizados a lo largo de visitas a las comunidades mencionadas. En el marco de estos encuentros presenciales se hizo uso de la entrevista estructurada como técnica, que se complementó con la observación participante.

Por lo expresado se utilizaron técnicas para recolectar datos como entrevistas, literatura, revisión de documentos periodísticos, anotaciones, grabaciones e interacción e introspección con grupos o comunidades, con las que se trató de descubrir, construir e interpretar determinadas realidades.

Si bien se estimó analizar las posibilidades de aplicación en el contexto centroamericano, se aprovechó la visita realizada a diferentes lugares de Costa Rica (setiembre de 2007) y demás países centroamericanos (de febrero a noviembre de 2008). En dichas oportunidades, no solo se aprovechó para recopilar información sino que se contó con la visita de especialistas europeos con los que se conversaron aspectos referentes al tema en estudio.

No obstante, Costa Rica fue la base en el que desarrollo el trabajo de campo, esto ya que es el único país de la región centroamericana que posee un reglamento específico relacionado con las denominaciones geográficas y en el cual se percibe una relativa articulación y resultados incipientes del uso y aplicación de estos instrumentos comerciales en algunas comunidades rurales, principalmente del sector caficultor y artesanal.

\section{Antecedentes}

Según defensores de la globalización económica, en el marco de apertura comercial presentada desde el ocaso del siglo XX, los mercados locales han ido perdiendo importancia para dar paso a un mercado global único. En ese contexto, las Denominaciones Geográficas Protegidas 
(DGP), se visualizan como instrumentos comerciales y signos de "valor agregado", que pueden proporcionar a productos determinados, mayor poder de venta en los mercados internacionales (Organización Mundial de la Propiedad Intelectual-OMPI, 2001).

De conformidad con la lógica referida, las Denominaciones Geográficas como instrumentos comerciales protegen productos con prestigio, mismo que radica de una u otra forma, en un origen geográfico y en elementos culturales propios; únicos, exclusivos y reconocibles. Ello implica que pueden utilizarse para cualquier producto que sea consecuencia de factores propios del lugar de origen, como clima, saber hacer, conocimientos y tradiciones de un pueblo, una ciudad, una región o un país. Para entender mejor la idea, sirve de ejemplo el término Switzerland (Suiza) o Swiss (suizo), considerado Denominación Geográfica Protegida (DGP), para los productos originados en Suiza, tal es el caso de los prestigiosos relojes suizos y sus chocolates (Organización Mundial de la Propiedad Intelectual-OMPI, 2001).

En esa dirección, no resulta casual, que la demanda de productos con distinciones geográficas protegidas haya logrado significar una actividad de gran crecimiento en la última década. Según López (1996), solo en Francia se registraba para el año 2000, casi 700 denominaciones de origen protegidas a nivel nacional e internacional, entre las que destacan los famosos Bordeaux, Cognac y Champagne. Pues para él,... "no es lo mismo tomar champagne que sidra de manzana" (López, 1996, p. 2).

Consecuentemente, se presentan intendencias crecientes al consumo de productos amparados con distinciones en países que hasta hace poco, no mostraban interés por su aplicación. Bien anota Hernández (2000) que éstas "han roto fronteras al proyectarse cada vez más hacia el exterior" (p. 20)
Rusia y China son ejemplo de ese fenómeno comercial y en la actualidad, participan al igual que otros países en desarrollar y consolidar las Denominaciones Geográficas; bajo el supuesto de que ayudan a valorar mejor sus productos y a estimular la economía rural. Li Dongsheng, Viceministro de la Administración Estatal de Industria y Comercio de China (SAIC), asegura que su país...

(...) está aplicando diversas políticas destinadas a promover el uso de las indicaciones geográficas como un medio para "acelerar la construcción de un nuevo agro socialismo"y desarrollar el comercio internacional. Las indicaciones geográficas ofrecen a los campesinos dedicados a la producción individual, que carecen de los recursos económicos y las capacidades para crear marcas, la posibilidad de compartir los beneficios de las marcas sin necesidad de crear una marca ni de producir en serie (Organización Mundial de la Propiedad Intelectual -OMPI, 2007, p. 24).

Con relación a lo anterior, la Organización Mundial de la Propiedad Intelectual (OMPI) señala, que desde la incorporación de las denominaciones geográficas en el sistema nacional de marcas de China, más de 250 productos dentro de esa modalidad se han registrado en ese país, y aún quedan pendientes centenares de solicitudes que aspiran por obtener dicha protección. El auge mostrado desde finales de 2004, lo atribuye a que:

(...) diferentes entidades gubernamentales han concertado campañas para difundir el conocimiento y el uso de las mismas entre los agricultores y las empresas de las zonas rurales. Esto se complementó con medidas jurídicas muy promocionadas, en el marco de las cuales los organismos encargados de la aplicación de la ley investigaron y procesaron judicialmente casi 300 infracciones contra, por ejemplo, el pickle en conserva de Fuling, el arroz de Xiaozhan y la pera aromática de Kurle (Organización Mundial de la Propiedad Intelectual-OMPI, 2007). 
Bajo esas primicias, se percibe que el desarrollo de las distinciones geográficas protegidas ha comenzado a expandirse por todas las regiones del planeta. Ante ello, resulta de suma importancia realizar un análisis de la viabilidad en cuanto al desarrollo y consolidación de estos instrumentos comerciales en Centroamérica. Principalmente, cuando se parte de que es una región con amplio patrimonio tangible e intangible y que se encuentra próximo, a entrar en vigencia el Acuerdo de Asociación entre la Unión Europea y la región; en el cual se establece ciertas prerrogativas de acceso, para productos con denominaciones geográficas protegidas.

\section{Viabilidad para implementar denominaciones geográficas protegidas en centroamérica}

Para Sánchez y Villalobos (2010), en la actualidad, la Unión Europea (UE) es el tercer socio comercial de la región centroamericana, misma con la que se espera intensificar relaciones comerciales una vez que el Acuerdo de Asociación tenga vigencia efectiva. Al respecto se refieren a que:

Desde 1998, el comercio entre Centroamérica y la Unión Europea se ha incrementado considerablemente. En ese año, el incremento comercial entre ambas regiones superó los 3700 millones de dólares ya que para 2008 había alcanzado los 6360 millones de dólares, lo que representó un crecimiento del $70 \%$ a una tasa promedio anual del 5,8\% (...) durante la mayor parte del periodo, se registró una balanza comercial positiva para la Unión Europea, sin embargo, en lo que respecta a las exportaciones, Centroamérica fue la que presentó las mayores tasas de crecimiento anual de las dos regiones $(11,4 \%$ y 10,6\% respectivamente) (p. 9-10).
En promedio, desde el año 2000, las exportaciones que dirigió Centroamérica a la Unión Europea representaron aproximadamente un 13\% de las exportaciones totales de la región Centroamericana mientras que las importaciones provenientes de esa zona representaron un 9\% del total importado. Lo trascendental de este entramado es la oferta exportable que presenta Centroamérica a esa región, pues del total de las exportaciones dirigidas a ese mercado, el $61.8 \%$ son mercancías clasificadas en el sector agrícola, de las cuales sobresalen exportaciones de café oro, bananas frescas, piñas, melón, entre otros, que acumulan una participación del 44.2\%; mientras que las importaciones provenientes de la UE tratan en su mayoría de bienes finales (Secretaria de Integración Económica Centroamericana-SIECA, 2006). El flujo comercial y abastecimiento de Centroamérica a la UE de ciertos productos agrícolas, se ilustra en siguientes cuadros.

De conformidad con la información presentada en la Tabla 1 y 2, se explica que para los centroamericanos el sector agropecuario representa aún, un porcentaje significativo del PIB nacional y constituye una de las principales actividades generadoras de empleo. En consideración, el comportamiento de los flujos comerciales con Europa es creciente, esto lo evidencia las cifras obtenidas para el 2005, año en que la tasa de crecimiento de las exportaciones regionales hacia ese destino alcanzó el 13,1\%, tal y como se muestra en el siguiente cuadro (Ministerio de Comercio Exterior de Costa Rica - COMEX, 2006).

En general, los datos obtenidos de la Secretaría de Integración Económica Centroamericana (SIECA) refieren que Costa Rica representa el 38,10\% de lo que exporta la región a la UE. No obstante, este panorama no exime a demás países de la región a visualizar a los 500 millones de consumidores comunitarios europeos, como mercado potencial para dirigir sus productos (Rivera, 2008). 
TABLA 1

Principales productos centroamericanos que se exportan a la Unión Europea (2005)

\begin{tabular}{|c|c|c|}
\hline PRODUCTOS & MILES US\$ & PARTIC. $\%$ \\
\hline Café sin tostar y sin descafeinar & 582837 & $30,0 \%$ \\
\hline Partes y accesorios de máquinas automáticas para el procesamiento de datos & 28128 & $11,7 \%$ \\
\hline Bananas o plátanos, frescos o secos & 22997 & $11,5 \%$ \\
\hline Piñas tropicales (ananás), frescas o secas & 69889 & $8,7 \%$ \\
\hline Equipos eléctricos para amplificación de sonido & 86990 & $4,5 \%$ \\
\hline Camarones, langostinos y demás Decápodos natantia, congelados & 69667 & $3,6 \%$ \\
\hline Follaje, hojas, ramas y demás partes de plantas, frescos & 56428 & $2,9 \%$ \\
\hline $\begin{array}{l}\text { Atunes, listados y bonitos (Sarda spp), enteros o en trozos (preparaciones } \\
\text { o conservas) }\end{array}$ & 51159 & $2,6 \%$ \\
\hline Melones frescos & 30853 & $1,6 \%$ \\
\hline Las demás plantas vivas & 30072 & $1,5 \%$ \\
\hline Minerales de cinc y sus concentrados & 20622 & $1,1 \%$ \\
\hline Las demás compotas, jaleas y mermeladas & 19539 & $1,0 \%$ \\
\hline $\begin{array}{l}\text { Antenas y reflectores de antena de cualquier tipo; partes apropiadas para utilizar con } \\
\text { dichos artículos }\end{array}$ & 17880 & $0,9 \%$ \\
\hline Cacahuates (maníes) sin tostar, sin cáscara & 9810 & $0,5 \%$ \\
\hline Ron y demás aguardientes de caña & 8607 & $0,4 \%$ \\
\hline Subtotal & 1605478 & $82,7 \%$ \\
\hline Otros productos & 336361 & $17,3 \%$ \\
\hline Total & 1941839 & $100,0 \%$ \\
\hline
\end{tabular}

Fuente: Secretaría de Integración Económica Centroamericana (SIECA), 2006. 
TABLA 2

Principales productos centroamericanos que se importan de la Unión Europea (2005)

\begin{tabular}{|c|c|c|}
\hline PRODUCTOS & MILES US\$ & PARTIC. $\%$ \\
\hline Circuitos integrados monolíticos, digitales & 96374 & $13,6 \%$ \\
\hline Otros medicamentos, dosificados o acondicionados para la venta al por menor & 174783 & $6,0 \%$ \\
\hline Aceites ligeros (livianos) y preparaciones, de petróleo o mineral bituminoso & 130053 & $4,4 \%$ \\
\hline $\begin{array}{l}\text { Los demás vehículos con motor de émbolo alternativo, de encendido por chispa, de cilin- } \\
\text { drada superior a 1,500 cm3 pero inferior o igual a } 3,000 \mathrm{~cm} 3\end{array}$ & 48657 & $1,7 \%$ \\
\hline $\begin{array}{l}\text { Los demás aparatos de telecomunicación por corriente portadora o telecomunicación } \\
\text { digital }\end{array}$ & 35971 & $1,2 \%$ \\
\hline $\begin{array}{l}\text { Aparatos emisores de radiotelefonía, radiotelegrafía, radiodifusión o tv, con } \\
\text { aparato receptor incorporado }\end{array}$ & 29794 & $1,0 \%$ \\
\hline $\begin{array}{l}\text { Los demás productos laminados planos, enrollados, simplemente laminados en caliente, } \\
\text { de espesor inferior a } 3 \mathrm{~mm}\end{array}$ & 23113 & $0,8 \%$ \\
\hline Los demás aceites de petróleo o mineral bituminoso, excepto aceites ligeros & 22063 & $0,8 \%$ \\
\hline Aparatos emisores de radiotelefonía, radiotelegrafía, radiodifusión o televisión & 21434 & $0,7 \%$ \\
\hline Fungicidas & 20515 & $0,7 \%$ \\
\hline Los demás medicamentos, sin dosificar ni acondicionar para la venta al por menor & 19652 & $0,7 \%$ \\
\hline Urea, incluso en disolución acuosa & 18188 & $0,6 \%$ \\
\hline Sulfato de amonio & 15925 & $0,5 \%$ \\
\hline Los demás aparatos de telefonía o telegrafía & 14973 & $0,5 \%$ \\
\hline $\begin{array}{l}\text { Los demás placas y baldosas, de cerámica, barnizadas o esmaltadas, para } \\
\text { pavimentar o revestir }\end{array}$ & 14553 & $0,5 \%$ \\
\hline Subtotal & 986048 & $33,7 \%$ \\
\hline Otros productos & 938949 & $66,3 \%$ \\
\hline Total & 2924997 & $100,0 \%$ \\
\hline
\end{tabular}

Fuente: Secretaría de Integración Económica Centroamericana (SIECA), 2006. 
TABLA 3

\section{Balanza Comercial de Centroamérica con la Unión Europea (1998 - 2005) En millones US\$}

\begin{tabular}{|l|c|c|c|c|c|c|c|c|}
\hline & $\mathbf{1 9 9 8}$ & $\mathbf{1 9 9 9}$ & $\mathbf{2 0 0 0}$ & $\mathbf{2 0 0 1}$ & $\mathbf{2 0 0 2}$ & $\mathbf{2 0 0 3}$ & $\mathbf{2 0 0 4}$ & $\mathbf{2 0 0 5}$ \\
\hline EXPORTACIONES & 2112,9 & 2136,2 & 1952,4 & 1360,3 & 1305,2 & 1571,7 & 1716,6 & $1.941,8$ \\
\hline Tasa de crecim. X (\%) & n.a. & $1,1 \%$ & $-8,6 \%$ & $-30,3 \%$ & $-4,0 \%$ & $20,4 \%$ & $9,2 \%$ & $13,1 \%$ \\
\hline IMPORTACIONES & 1601,3 & 1522,8 & 1574,6 & 1783,7 & 2027,5 & 2347,0 & 2607,9 & $2.925,0$ \\
\hline Tasa de crecim. M (\%) & n.a. & $-4,9 \%$ & $3,4 \%$ & $13,3 \%$ & $13,7 \%$ & $15,8 \%$ & $11,1 \%$ & $12,2 \%$ \\
\hline SALDO & 511,6 & 613,4 & 377,8 & $-423,4$ & $-722,3$ & $-775,3$ & $-891,3$ & $-983,2$ \\
\hline $\begin{array}{l}\text { Tasa de crecim. (X-M) } \\
\text { (\%) }\end{array}$ & n.a. & $19,9 \%$ & $-38,4 \%$ & $-212,1 \%$ & $70,6 \%$ & $7,3 \%$ & $15,0 \%$ & $10,3 \%$ \\
\hline
\end{tabular}

Fuente: Secretaría de Integración Económica Centroamericana (SIECA), 2006.

Así es necesario cada vez más, intensificar esfuerzos para complacer en lo posible a este grupo de consumidores, que leen con detenimiento la información que se brindan en las etiquetas de los productos, que analiza los elementos que lo conforman, la procedencia de la materia prima y el nombre de la empresa que los elaboró y distribuyó. No cabe duda, que de esto depende la decisión de la compra, pues para este resulta importante, saber con exactitud la procedencia y detalles de lo que consumen (Camacho, 2008).

Atendiendo a ello, en el ámbito centroamericano se perciben incipientes innovaciones, contactos con instituciones de gobierno, firmas extranjeras y nacionales y de cooperación internacional interesadas en impulsar y desarrollar las distinciones geográficas. Las mismas se concretan en apoyos a productores decididos a llevar mejoras a las prácticas de producción y mantener técnicas de cultivo tradicional, principalmente pequeños productores que años atrás, se encontraban en bancarrota y sin más, que abandonar los lugares de origen (Barquero, 2003).

Por consiguiente, Echandi (2008) anota que se ha incrementado en el istmo, programas para maximizar oportunidades de exportación, que persiguen mejorar y sistematizar las prácticas de producción, manteniendo el respeto por la biodiversidad, el rescate de los suelos y la preservación del ambiente, y la tradición. Resultados concretos de esas acciones destaca la promoción y aceptación de una serie de productos en el exterior, a los que algunos aducen, que se les paga por "el costo del sabor" hasta $40 \%$ sobre el precio regular.

Aunque estas referencias no son exactamente ejemplos de Denominaciones Geográficas y tratan en realidad de marcas colectivas con connotación geográfica, vale indicar que la mención del origen ha permitido mejor colocación y venta de los productos. Particularmente, como señala Barquero (2003), ya más del 50\% de la producción nacional de café se logra exportar bajo condición de "fino y de primera calidad", y alrededor del $85 \%$ obtienen precios superiores a los de la bolsa, por lo que la puesta en marcha de algunas de las acciones se ha convertido en ventajas competitivas para el istmo, ya que proporcionan un sobreprecio al productor en pago a la calidad. 
Ahora, es claro que para los productores el uso de estas herramientas se puede traducir en elementos de promoción, valorización de recursos locales, estrategia de desarrollo rural y plataforma de apoyo para empresas pequeñas y medianas locales; sin embargo, especialistas insisten que sean los propios productores los que gestionen la protección de las denominaciones geográficas (Barquero, 2003).

Por tanto, los contactos e injerencia de grupos externos en el proceso deben ser vistos sigilosamente, pues los productos pueden ser afectados ante la ofensiva de ajenos que pueden transgredir, falsear y abusar de la reputación de productos locales, confundiendo así al consumidor sobre el origen de los mismos. Al respecto, la Organización Internacional de Indicaciones Geográficas (ORIGIN, por sus siglas en inglés) promueve que sean las mismas comunidades las que tengan control de sus denominaciones, y las que deben en consenso, proponer, diseñar, difundir e implementarlas para su éxito.

Esa idea conlleva a reforzar la larga tradición de asociacionismo de productores en Centroamérica, lo cual se presenta en una fortaleza para la implementación de una Denominación de Origen Protegida. La presencia de numerosas cooperativas incide y ha desempeñado un papel fundamental como dinamizadoras de la actividad productiva en comunidades rurales. Pues los productores reciben capacitaciones específicas para preservar y mejorar la calidad del producto, pueden acceder a créditos que les permiten brindar un manejo más óptimo a sus cultivos o renovarlos y los precios recibidos por la venta a la cooperativa son mayores que en el caso de vender directamente a intermediarios o son negociados por los propios productores (González, s.f.).

\section{Limitaciones y estrategias para implementar las DGP}

En general existe una percepción positiva acerca de las ventajas de proteger los nombres de los lugares donde se originan los productos. No obstante, se presentan factores limitantes para establecerlas de forma adecuada en la región, que de superarse pueden constituir una excelente manera, para incidir en el desarrollo local y acceder en mejores condiciones a nuevos nichos de mercado (García, 2001).

En concreto, se mantiene un relativo desconocimiento sobre el tema al no conceptualizarse integralmente, tanto en instituciones públicas como sectores productivos, lo que insta a incentivar procesos educativos con actores del sector público, privado, productores, fabricantes y comunidades en general; igualmente el desarrollo de acciones que promocionen la cultura de reconocimiento (Granados, 2004).

En atención a esas carencias se han generado algunas iniciativas patrocinadas por Agencias de Cooperación Internacional dirigidas principalmente a caficultores y técnicos. Éstas buscan fomentar el conocimiento del sistema de Denominaciones de Origen en las entidades oficiales y privadas, para su puesta en funcionamiento. La idea es aplicar el sistema de calidad diferenciada al ámbito específico del café, teniendo en cuenta sus especificidades técnicas, legales y comerciales, además que conozcan las diferencias legales existentes entre el sistema europeo y centroamericano, así como sus repercusiones en la Organización Mundial del Comercio (Proyecto Alianza de Aprendizaje, 2008).

Por ende, para establecer exitosamente un sistema de distinciones geográficas se recomienda involucrar diferentes áreas de actividad en el orden económico, comercial, social, cultural, histórico, jurídico, normalización, certificación, acreditación, sistemas de calidad, entre otros, 
que intervienen en el proceso. Según Sergio Navas, Vicepresidente Ejecutivo de la Cámara de Exportadores de Costa Rica (CADEXCO): "Este indicador crea mayor competitividad, pero no se ha explotado por falta de administración del comercio y asesoría al pequeño y mediano productor" (González, 2006, p. 12).

Haciendo eco de lo anterior, resulta necesario habilitar estructuras que coordinen y gestionen la operación del sistema, a saber, Secretarías Técnicas y Consejos de Denominación de Origen, que ayuden a fijar directrices en materia de política de calidad y promoción de los alimentos. Razones que invitan a valorar la conveniencia de trasladar a instancias superiores la necesidad de firmar acuerdos internacionales de protección en la materia (González, 2008).

Entre otras acciones sugeridas se percibe el desarrollo de instrumentos efectivos de apoyo y orientación para el estudio de productos, la elaboración de guías para la identificación de productos con distinción y la elaboración de pliegos de condiciones, lo mismo que formularios para los trámites de inscripción y registro adecuados.

Otro aspecto pendiente es la determinación de los costos para desarrollarlas en la región, pues la implementación requiere de costos altos para mejorar procesos de tecnología, estandarizar los métodos y del producto, así como establecer un órgano de control financieramente autosuficiente, que debería compensarse contra los beneficios esperados (Comunidad Autónoma de Galicia, 2005).

Ante esas realidades y la de no contar con subsidios de la magnitud europea, a las diferentes agrupaciones de productores les corresponde desarrollar iniciativas asociativas y empresariales para conformar fondos, que ayuden a garantizar condiciones comunes y controladas para los productos comercializados con la denominación o sello respectivo. En caso contrario verían perder cuota en el mercado y la presencia de los productos sustituidos en la práctica, por otro producto distinto con el mismo nombre (Asociación Reguladora de las Normas de la Denominación de Origen de los Santos, 2008).

Concienciar a los productores beneficiados es entonces tarea crucial, por lo que deben elaborar un plan de información que convenza a los demás con la difusión de experiencias exitosas, utilizando todos los medios de comunicación comerciales y culturales de la zona, entre otros disponibles. Figura como ejemplo, estrategias empleadas por los caficultores de la zona de los Santos (Costa Rica), en la que a través de los medios de comunicación, incentivan y concientizan a los productores para la producción de café de calidad, con la consecuente mejoría de las condiciones de vida de sus comunidades. Los espacios también se aprovechan para convocar a reuniones y capitaciones a productores involucrados (Asociación Reguladora de las Normas de la Denominación de Origen de los Santos, 2008).

Por ejemplo, en la actualidad la cantidad de café que se recibe en el beneficio de Coopedota es de 65000 fanegas anuales. El aumento se debe entre otras razones, al mejoramiento continuo que aporta a sus asociados en el campo tecnológico y agrícola. La misma persigue mejorar la calidad del café, llegando a premiar a los productores.

En fin, estas estrategias han logrado resultados satisfactorios, entre ellas hacer partícipes desde el principio, a pequeños productores y cooperativas para evitar la exclusión así como concientizar sobre los beneficios que las denominaciones geográficas pueden traer. Así se vislumbra como acción importante, el incentivo de valores como solidaridad, responsabilidad y el asociacionismo, para que aumente la integración en la cadena de comercialización. Además se involucra el valor de la tradición del cultivo mediante la historia y tradiciones de la caficultura en el área, con la participación de todos los productores. 
Destaca entre otras estrategias implementadas por los caficultores de la zona, el uso de símbolos, que avocan elementos de cohesión e identidad como región, en donde se realza elementos propios del folclor, manifestaciones artísticas como poemas y canciones alusivas a las denominaciones que las vinculan como parte del patrimonio intangible de sus comunidades. Bajo el lema "todo cuenta", se propaga el uso de signos externos en negocios, instituciones, casas y vehículos (Asociación Reguladora de las Normas de la Denominación de Origen de los Santos, 2008).

Así reza una canción del caficultor (doteño), Luis Fernando Chacón Monge (2008), quién compuso a la denominación de origen Tarrazú. La misma exalta la calidad del café, concientiza a los productores de la influencia del origen del café en su particular calidad y la necesidad de protegerlo, por lo que alerta la competencia desleal y el engaño que pueden sufrir los consumidores. La canción se suele escuchar en programas de la emisora local, Radio Cultural Los Santos. Entre sus estribillos destaca las siguientes frases.

(...) Nos quieren robar el nombre, aunque no sean ni vecinos, pues son muchos los intereses de unos pocos que son vivos, no se saldrán con las suyas mientras estemos $\operatorname{vivos}(\ldots)$

Si somos muy diferentes con orgullo lo deci$\operatorname{mos}(\ldots)$

Si lo nuestro es nuestro, no sean tan aprovechados y al final el consumidor no se sentirá engañado (...)

\section{Condiciones jurídicas}

Desde hace dos décadas se habla de libre comercio en la región, contexto en que las naciones centroamericanas han pasado a ser parte de numerosos instrumentos internacionales que rigen el tema de las indicaciones geográ- ficas. Destacan el Acuerdo Marco de Cooperación entre la Comunidad Económica Europea y las Repúblicas Centroamericanas, el cual hace referencia a normalizar, certificar la calidad y la protección recíproca de las denominaciones de origen (Convenio Centroamericano para la Protección de la Propiedad Industrial, 1970).

Entre otros, el Convenio Centroamericano para la Protección de la Propiedad Intelectual-CCPPI (1970), que dedica el Título VI (capítulo Único) a las Indicaciones de Procedencia y de las Denominaciones de Origen. En el artículo 72, párrafo segundo, define como denominación de origen: "(...)al nombre geográfico con que se designa un producto fabricado, elaborado, cosechado o extraído en el lugar al cual corresponde el nombre usado como denominación y que sirve para determinar una calidad y ciertas características".

Otro importante instrumento jurídico que se adiciona a la lista, son los Acuerdos de los Aspectos de Propiedad Intelectual relacionadas con el comercio (ADPIC, por sus siglas en inglés), de la Organización Mundial del Comercio (OMC), mismo que permite a Centroamérica proteger las denominaciones. También existe el Arreglo de Lisboa y las Leyes de Marcas y Otros Distintivos aprobadas en algunos países de la región.

Según Correa (1996), la aplicación de estos instrumentos jurídicos supondría que las distinciones geográficas hubiesen sido respetadas en el comercio internacional, al estar en sintonía con los estándares mínimos dispuestos en el ADPIC y con la política de calidad de la UE y de la OMC; por sí mismos, no aportan elementos suficientes para un adecuado desarrollo de las distinciones geográficas protegidas, ante la ausencia de reglamentos e institucionalización apropiada para el registro de este tipo de derechos en Centroamérica.

No obstante, las nuevas tendencias presentadas en los últimos años, han impulsado cambios sustantivos en el ordenamiento jurídico interno de 
la región, para que la gran variedad de productos puedan registrarse con denominación geográfica. Por lo que órganos de control nacional y regional, agricultores y cooperativas de productores, han sumado esfuerzos para proteger el patrimonio común constituido por el prestigio de un nombre geográfico determinado.

Más que aspectos para la operación y regulación adecuada de un sistema de indicación geográfica, lo anterior se debe entender como un conjunto de acciones pendientes, que han sido tomadas en cuenta recientemente, para evitar posibles contradicciones en las relaciones comerciales con otras regiones a las que destinan sus productos (González, 2008).

Pese a los esfuerzos dirigidos en la materia, según un estudio realizado en años recientes por Granados (2004), persisten condiciones que distan mucho de implementar una operación y regulación de un sistema de indicación geográfica adecuada. Grosso modo, para él persiste la idea de entender las denominaciones geográficas como signos distintivos incluidos dentro de las leyes de marcas en los países centroamericanos. Por lo que es común, que estén regidas e integradas en muchos aspectos en el marco dentro de esas disposiciones, lo cual es contrario en la UE, donde se regulan por una legislación independiente.

Asimismo, otras críticas que surgen y quizás la más importante, es que las disposiciones no hacen referencia directa a los métodos o procesos de transformación o elaboración del producto; se omite el grado de detalle en que deben ser descritas las cualidades, el carácter tradicional, o las características de prestigio y renombre del producto, así tampoco establecen estructuras de control específicas para los productos registrados.

Lo antepuesto se adiciona, a la variedad de conceptos legales formulados por los estados centroamericanos para la protección de las de- nominaciones geográficas, basados en sus variaciones legales, históricas y condiciones económicas locales. Ante esas circunstancias, se presenta disparidad y tantas excepciones que resultarían eventualmente inútiles de aplicar en la práctica (Gutiérrez, s.f).

Aunque en el tema de indicaciones geográficas la UE continúa a la vanguardia, lo cierto es que Centroamérica ha dado algunos pasos importantes en este sentido. En particular, Costa Rica introdujo el primer marco normativo sobre denominaciones de origen e indicaciones geográficas a través de la Ley de Marcas y Otros Signos Distintivos, Ley n. 7978 , del 6 de enero de 2000. La ley introdujo en su oportunidad, elementos innovadores, tanto por requerimiento del ADPIC (Acuerdo sobre los Aspectos de los Derechos de Propiedad Intelectual Relacionados con el Comercio), como por influencia de otras legislaciones (Castro, 2000).

Para Montero (2007), lo trascendental de esta legislación fue que vino a sustituir el viejo Convenio Centroamericano para la Protección de la Propiedad Industrial, rector de la materia en varios países centroamericanos por más de 30 años. Sin embargo, esto no bastó y quedaron pendientes normas complementarias que reglamentaran de manera específica la aplicación, definición y regulación de las denominaciones de origen e indicaciones geográficas. El vacío legal se mantuvo hasta la promulgación de un reglamento que derivara disposiciones relativas a las denominaciones de origen, mismo que se aprobó en noviembre de 2007, como Reglamento de las Disposiciones Relativas a las Indicaciones Geográficas y Denominaciones de Origen, que dedica un capítulo a definir las características de los bienes que recibirán la denominación y quiénes podrán participar. La norma permite luego de la inscripción, que los productos procedentes en una zona geográfica delimitada puedan identificarse con el nombre de la zona. 
Otros esfuerzos reconocidos a nivel centroamericano son los realizados por Honduras, que recibieron un respaldo significativo en 2004, cuando para cumplir con el Tratado de Libre Comercio entre Estados Unidos y Centroamérica (CAFTA), reformó sus leyes de propiedad intelectual y estableció un sistema para el registro y protección de los indicadores y denominaciones de origen. Pese que el segundo no fue requerido por el tratado, se fortaleció la infraestructura de la propiedad intelectual (Productores Agropecuarios Orgánicos de Juáres- Organicoop, 2007).

En términos generales, los países centroamericanos, a excepción de Costa Rica, continúan sin reglamento, la legislación es limitada, no aporta elementos suficientes que permitan establecer desde ese ámbito un adecuado desarrollo de distinciones geográficas que incluya el diseño de una estructura general de organización para su operatividad.

Por consiguiente y basados en la experiencia europea, el tema debe contemplarse cuando discusiones internacionales en materia de propiedad intelectual, comercio, lo mismo que convenios y tratados bi y multinacionales, incluyen elementos que regulan las condiciones de uso de los nombres geográficos, con el fin de evitar posteriores conflictos y obstáculos a la libre circulación de mercancías. En este contexto, lo importante no es solo crear reglamentos que actúen en el mercado nacional, sino que las indicaciones geográficas sean reconocidas en el mercado europeo y den valor agregado a los productos de exportación (Comunidad Autónoma de Galicia, 93, 2005).

Al respecto, Granados (2004) insiste que más que un marco jurídico e institucional adecuado, para el desarrollo y consolidación del sistema, resulta fundamental, incluirlas como parte de la política general de desarrollo rural y de mejoramiento de la calidad agroalimentaria e integrar así, el tema a las políticas y programas del sector agropecuario de la región.

\section{Productos centroamericanos con potencial a optar distinciones geográficas protegidas}

Después de la independencia de España en 1821, las nuevas naciones centroamericanas enfrentaron el dilema de buscar un modelo de desarrollo económico alternativo, que permitiera el despegue e inserción de la región al mercado mundial. Según Samper (1991) luego de varios intentos con productos de reminiscencia colonial como el añil, el cacao y el tabaco, el café logró tan ansiado cometido.

A partir de 1840, la historia de Centroamérica se comienza a escribir de otra manera, no solamente por el crecimiento económico al que se asocia sino por los efectos que causa en la población rural. En particular, el auge económico y la cultura de comercialización se tejió alrededor del café, conformó bases de identidad nacional, redefiniendo el paisaje de los campos e incipientes ciudades, reconfigurando el uso del tiempo en función de sus ciclos productivos (Arias \& Vives, 2005).

En el contexto actual del comercio y las tendencias en la oferta y la demanda, la producción de cafés diferenciados, entre ellos con origen específico, se presenta como elemento favorecedor para el acceso al mercado. Esto resulta mucho más atractivo cuando, según los datos, después del petróleo, el café se convierte en el producto más importante en el comercio internacional (Castillo, 2007).

A la vista de los resultados presentados, el café se percibe como los "Viñedos de Centroamérica”, tal apreciación se fundamenta por ser el producto con mayores características potenciales para ostentar la denominación geográfica, al tener productores concienciados de la importancia de 
la calidad del café para su economía, al constituir un producto en el que existe un elevado número de productores asociados en cooperativas o pequeñas empresas rurales que mantienen prácticas culturales relativamente homogéneas, además que la ubicación geográfica en que se produce es excepcional (suelo, clima y altitud) (Hall,1991).

Además del café y banano, figuran una serie de productos propios de un clima tropical, como el pejibaye, piña, granos, raíces y tubérculos, frutas frescas y secas, mieles, quesos, carnes, azúcar y conservas, que por su potencial pueden optar por distinciones geográficas protegidas. En la lista se incluyen productos tradicionales preparados como tamales, panes, rosquillas y bizcochos, jaleas y cajetas, tortillas caseras, entre otros, que son parte de las prácticas culturales centroamericanas (Granados, 2004).

Sin duda, todos los argumentos justifican la implementación de un sistema de distinciones geográficas en el ámbito regional con facilidad, más cuando existe abundante patrimonio de recursos naturales, históricos y culturales, conformado por una amplia gama de productos agrícolas, alimenticios y artesanales, que en el tiempo han venido a representar símbolos de identidad regional y que sus pobladores se consideran "dueños legítimos" (Chacón, 2008).

Consecuentemente hasta no realizar estudios más específicos, los mismos serán entendidos solo como productos con "potencial". Es imprescindible entonces elaborar listas de productos con potencialidad, considerando criterios propios como origen, tradición, características diferenciales, reputación, territorio, criterios de tipo económico y beneficios comerciales en el mercado nacional e internacional, como una base que dirigiría los esfuerzos para el desarrollo de las distinciones de los productos.

\section{Denominaciones de origen centroamericanas}

\section{Costa Rica}

El café se refiere al producto con mayor potencial para ostentar signos distintivos geográficos. Varios productores ya han logrado evitar a los intermediarios tradicionales y vender directamente el total de su producción a los puntos de venta. Con este esquema, pequeños productores de Santa María de Dota, San Marcos de Tarrazú, entre otras zonas cafetaleras, han logrado mejores precios de venta. Para el año 2006, el pago por quintal (46 kilogramos) que rondaba era de US\$170 al agricultor (Morales, 2006).

Por la importancia económica y el prestigio ampliamente reconocido del café, a finales de 2003, la Agencia Española de Cooperación Internacional (AECI), brindó asistencia técnica en aquellos temas en los cuales se podía mejorar las oportunidades de vida de los productores cafetaleros rurales, especialmente en Denominaciones de Origen y Agroturismo Cafetalero. La actividad fue apoyada con expertos en vinos de reconocida calidad mundial de la UE y miembros del Consejo Regulador de la Denominación de Origen Calificada de la Rioja de España (Instituto del Café de Costa Rica-ICAFE, 2004).

Bajo esta filosofía, el Instituto del Café (ICAFE) planteó a la Cooperación Española dos novedosos proyectos piloto como una manera de diversificar los ingresos de los productores, tomando como modelos las regiones de Los Santos y Orosí. Regiones que en el año 2000, el ICAFE había desarrollado una investigación muy pormenorizada sobre los aspectos distintivos de la calidad del café, los aspectos culturales e históricos, así como otros que deben ser caracterizados para avanzar hacia las denominaciones de origen (Instituto del Café de Costa Rica-ICAFE, 2004). 
Atendiendo a estos antecedentes, grupos organizados de productores se comenzaron a preparar para ostentar alguna denominación geográfica. En particular, iniciaron investigaciones históricas de sus productos y estudiaron los aspectos técnicos para tramitar los registros correspondientes sobre denominación de origen ante el Registro de la Propiedad Intelectual.

Las gestiones realizadas por la Asociación Reguladora de Normas de Denominación de Origen de Los Santos, conformada en 2005, son claro ejemplo de un grupo importante de caficultores interesados en proteger el prestigio alcanzado por el café, ante la ofensiva de agentes externos que pululan en la zona. Más que la obtención de la denominación, el fin de esta agrupación persigue proteger a sus cerca de 7.000 caficultores, ubicados geográficamente en las márgenes del río Pirrís, específicamente, en cantones de Dota, Tarrazú y León Cortés. Comunidades con fuertes lazos afectivos históricos, culturales y productivos, que la convierten en una unidad geográfica-culturaleconómica homogénea y diferente a otras zonas del país (Asociación Reguladora de las Normas de la Denominación de Origen de los Santos, 2008).

No es casualidad entonces que surjan en este contexto, más de una decena de grupos ajenos interesados en adquirir la codiciada denominación Tarrazú. La lucha de este grupo de caficultores se presenta ardua ante la ofensiva de intereses ajenos, que amplían en sus proyectos de pliego de condiciones la zona referenciada. De aceptarse diferentes delimitaciones, otras comunidades se incluirían en la denominación, a lo que la Asociación mencionada se opone rotundamente, ya que según la catación y los análisis de suelo indican que la calidad del producto no es la que ellos pretenden proteger (Asociación Reguladora de las Normas de la Denominación de Origen de los Santos, 2008).

En particular, la Asociación se encuentra sumamente organizada y convencida de forma- lizar y proteger el registro de la denominación geográfica de café Tarrazú, argumentado en su pliego de condiciones, que la zona en cuestión presenta una riqueza geográfica y edafoclimática con particularidades culturales y sociales, expresadas en modos de producción y elaboración propios que acumulan más de cien años. Según Mata (2008)..."Nosotros somos los dueños legítimos...tenemos que defender nuestro apellido... ese es nuestro origen."

Aproximadamente el 78\% de la superficie del cantón de Dota, la constituye la Reserva Forestal Los Santos, y un 5\% la Zona Protectora Cerro El Nara; esta última se ubica al sur de la región. En la parte central se localiza una franja de mineralización latérico férrica, y al norte una zona de mineralización preferente cuprífera con oro, hierro, zinc y plata. Desde los albores, los Marienses son caficultores 100\%. La calidad del café de la zona está catalogada por algunos como uno de los mejores del mundo.

Las particularidades comunes de Costa Rica con respecto a los países de la región, favorecen la diversidad de productos con formas y grados de vinculación al territorio. En razón, diversas opiniones en Costa Rica no solo se refieren al café, sino también a la importancia de desarrollar signos distintivos geográficos que pueden beneficiar otros productos catalogados como especiales (El Financiero, 2006).

Entre ellos figuran el queso de Turrialba, natilla de Zarcero, el pejibaye de Turrúcares, los muebles de Palmares, las toronjas rellenas de Atenas, entre otros; pues una denominación de origen ayudaría a estos productos a venderlos a un precio mayor. Figura también, la tradicional carreta típica Sarchíceña, la artesanía precolombina de San Vicente de Nicoya, ya que en esos casos se encuentra una serie de saberes ancestrales que dan por resultado un producto particular que identifica a la comunidad. De esta forma, es posible visualizarla como una actividad vigente, la 
cual se ha convertido en un importante mecanismo de subsistencia de una comunidad entera, que se aferra a su identidad (Weil, 1995).

Al igual que otros productores, la Asociación de Productores Agropecuarios de Santa Cruz de Turrialba (ASPROA) que agrupa a más de 275 productores de leche y fabricantes de queso, ha realizado desde hace varios años, gestiones importantes al respecto. En particular, las gestiones buscan que se califique a Turrialba como la cuna del queso, considerando que la producción en este cantón tiene una historia de más de 135 años y alcanza los 250.000 kilos de queso maduro y frescos por mes. La petición ante el Registro de Propiedad Intelectual incluye cuatro tipos de quesos, todos bajo la categoría de "queso Turrialba": fresco, maduro, fresco artesanado y maduro artesanado (Camacho, 2008).

Con el fin de lograr su cometido, han contado con el apoyo de varias instituciones públicas, para optar por esta herramienta internacional que les permitirá proteger el origen del queso turrialbeño y competir con socios comerciales de la UE, que ya tiene quesos protegidos como sucede con el manchego, mozzarella, parmesano, cheddar y el gouda.

Los apoyos se han dirigido a realizar estudios sobre la caracterización física, química, sensorial, de fincas y sus alturas, razas de ganado, aspectos históricos, de tradición, límites geográficos y del consumidor, dado que el reglamento incorpora dos temas fundamentales: el pliego de condiciones que regula lo relativo a estudios técnicos, del producto, materias primas, métodos de fabricación, factores humanos y naturales que acreditan el vínculo geográfico, entre otros (Camacho, 2008).

Por consiguiente, son varias zonas del país que podrían hacerse merecedoras de denominaciones de origen. Todo dependerá del esfuerzo que hagan por demostrar que realmente la producción de ciertos bienes o servicios son originarios de esos lugares.
TABLA 4

\section{Costa Rica Exportaciones de algunos productos potenciales a obtener Denominaciones Geográficas Protegidas (2010)}

\begin{tabular}{|l|c|}
\hline \multicolumn{1}{|c|}{ PRODUCTOS } & MILES US\$ \\
\hline Café sin tostar y sin descafeinar & 258807681 \\
\hline Bananas o plátanos, frescos o secos & 741870564 \\
\hline $\begin{array}{l}\text { Piñas tropicales (ananás), } \\
\text { frescas o secas }\end{array}$ & 666990736 \\
\hline Melones frescos & 73472724 \\
\hline Raíces de yuca & 524234449 \\
\hline
\end{tabular}

Fuente: COMEX con base en cifras de PROCOMER, 2010.

\section{Guatemala}

El café desempeña un papel crucial en la economía agrícola y en la dinámica del empleo de Guatemala, agrupa unos 65,000 productores y emplea al $11 \%$ de la población activa total del país (y temporal a cerca de un millón), cifras muy superiores a todos los otros sectores agrícolas de exportación. Es decir, que por las características del cultivo, las zonas donde se cultiva y la población que las habita, determina el núcleo de la dinámica económica nacional, siendo el cultivo que concentra en su ámbito la mayor cantidad de población más distribuida en el territorio nacional (Camacho \& Gilles, 1992).

La acidez refinada, textura achocolatada, cuerpo fino y pronunciado y el origen es parte fundamental para los cafés gourmet de Guatemala, por lo que se considera el producto con mayores potencialidades de adquirir denominación de origen. Estos elementos han sido considerados para la gigante estadounidense del café Starbucks, de ahí que este país se ha convertido en uno de sus principales proveedores. Tan importante es el origen del producto que el café ha construido un negocio muy exitoso alrededor de una idea muy sencilla "la geografía es un sabor" (Camacho \& Gilles, 1992). 
En particular, hace suponer que todo está listo para que a la región de Antigua le sea otorgado la Denominación, el proceso de certificación se encuentra en la fase legal y ya fue integrada la Asociación de Productores de Café Antigua (APCA). Además de la región de Antigua sobresalen las características especiales del café de Oriente, Fraijanes, Atitlán, Cobán, San Marcos y Acatenango (Dardón, 2006).

Para lograr estas calidades, la Asociación Nacional de Productores de Café (ANACAFE), ha identificando esas regiones con personalidad única, moldeada a partir de la altura, el microclima, el tipo de suelo y la lluvia. La regionalización surge de las estrategias de geoposicionamiento (identificación geográfica) y de certificación de la denominación de origen de todas las regiones de café que posee Guatemala.

Si bien, en Guatemala el café se ha perfilado como el producto con mayor potencial para ostentar una denominación protegida, se ha dilucidado la posibilidad y el interés por defender la originalidad colonial de la ciudad de Antigua, proteger ciertos rones y la variedad de trajes típicos de los diferentes grupos étnicos indígenas que también gozan de fama y prestigio nacional e internacional (Dardón, 2006).

\section{TABLA 5}

\section{Guatemala Exportaciones de algunos productos potenciales a obtener Denominaciones Geográficas Protegidas (2010)}

\begin{tabular}{|l|c|}
\hline \multicolumn{1}{|c|}{ PRODUCTOS } & MILES US\$ \\
\hline Azúcar & 726000100 \\
\hline Café sin tostar y sin descafeinar & 713882700 \\
\hline $\begin{array}{l}\text { Bananas o plátanos, frescos } \\
\text { o secos }\end{array}$ & 353175500 \\
\hline Materiales textiles (tejidos o telas) & 182898600 \\
\hline $\begin{array}{l}\text { Frutas frescas, secas o } \\
\text { congeladas }\end{array}$ & 166577900 \\
\hline
\end{tabular}

Fuente: Ministerio de Economía de Guatemala, 2010.

\section{Honduras}

El café hondureño ha sido solicitado por consumidores gourmet europeos durante décadas, pese a que los estándares de calidad de la producción no han sido altos históricamente. Por ello, se comercializó en los mercados mundiales con un descuento significativo en comparación con el café de sus vecinos Guatemala y Costa Rica. No obstante, el café se ha convertido en una de las pocas grandes historias de éxito de las exportaciones hondureñas en los últimos años. Su valor se ha triplicado desde 2001, los datos muestran que el valor creció un 16\% llegando a US\$ 470 millones, superando el récord anterior de US\$ 430 millones establecido en 1998, año en que Honduras fue devastado por el huracán Mitch (Productores Agropecuarios Orgánicos de Juáres- Organicoop, 2007).

Este repunte se debe entre otras razones, al esfuerzo de productores para ascender en la cadena de valores por medio de varios esquemas de certificación, con un mercado destino de consumidores altamente conscientes de la calidad y la responsabilidad social. Para ellos, la Denominación de Origen constituyó el mecanismo que mejor garantiza y protege la calidad del café, asimismo, la herramienta idónea para mejorar la competitividad y capacidad de penetración de nuevos mercados.

Para la ejecución de un proyecto de Denominación de Origen, se escogió la región cafetalera Marcala debido al prestigio reconocido en el mercado y a las características agro-ecológicas irrepetibles en el mundo. En 2005, el Café de Marcala se convirtió en la primera Denominación de Origen y única hasta el momento oficialmente registrada en Centroamérica. Para su reconocimiento participaron productores, tostadores, comercializadores y exportadores de la cordillera de Montecillos, que durante tres años trabajaron conjuntamente con apoyo técnico y financiero del Instituto Hondureño del Café (IH(AFE) y de la Agencia Española de Cooperación Internacional (AECI) (La Tribuna, 2008). 
Considerando que supone una experiencia piloto en Centroamérica, muchos son los logros que se han alcanzado desde 2005. Concretamente, más de 1,500 productores participaron en el proyecto con una población beneficiaria de aproximadamente 8,000 familias conformada por pequeños agricultores cafetaleros, ubicadas en 202 aldeas de 19 municipios pertenecientes a los departamentos La Paz, Comayagua y de Intibucá (Productores Agropecuarios Orgánicos de Juáres- Organicoop, 2007).

En particular, las Cooperativa Raos, la Coordinadora de Mujeres Campesinas de La Paz (COMUCAP), Café Orgánico Marcala Sociedad Anónima (COMSA) y Café Orovila fueron pioneras en lograr estos niveles de exportaciones. Uno de los principales retos fue lograr la exportación de los primeros lotes certificados con el sello DO en 2007, acción importante para dar a conocer la operatividad y funcionamiento de la Denominación, además de medir la respuesta del mercado internacional del café a la aceptación de ese signo distintivo. El 80\% de los lotes fueron exportados a la EU y el 20\% restante a los Estados Unidos de América, lo que confirma que el mercado potencial para Marcala es la UE, en particular el mercado Alemán (Productores Agropecuarios Orgánicos de Juáres- Organicoop, 2007).

Si bien Honduras obtuvo la primera Denominación Geográfica de café de Centroamérica, esto no significa que el reconocimiento este homologado en la UE, para eso se continúa trabajando para ostentarlo en esa región.

\section{Nicaragua y El Salvador}

Ciertamente, el resto de países llevan ventaja en la exportación de café fino, sin embargo, El Salvador y Nicaragua han dado pasos importantes. El Salvador ejemplifica el avance de la región en grano de calidad. De la cosecha de 2000-2001 a la de 2006-2007, la producción de café especial se quintuplicó, los productores han comprendido que normas como esas llaman la atención de las empresas compradoras y aumentan el nivel de la productividad (Castillo, 2007)
Pese a que se han dado esfuerzos importantes por obtener certificaciones y en destacar elementos diferenciadores de su café, según el Consejo Salvadoreño del Café, entre 2000 y 2004, la producción y las exportaciones cayeron más de un 40\%, lo que sumió a los cafetaleros en la deuda más elevada por quintal producido de la región (Castillo, 2007).

En general, el café de estos países no goza de la misma imagen que han desarrollado y poseen sus vecinos Guatemala y Costa Rica, que acumulan los diferenciales de precios más altos de la región. Asimismo, no se encuentran en iguales condiciones en el marco de las posibilidades de crear una denominación de origen para un determinado producto, en especial para el café. Dichas condiciones ponen de alguna manera en desventaja el café que ellos producen (Castillo, 2007).

Por otro lado, se menciona algunos productos con potencial para obtener Denominaciones de Origen Protegidas, en especial el "Bálsamo de El Salvador", por estar compuesto, principalmente, por un aceite llamado cinameina que se extrae del árbol de bálsamo, que es original de ese país. El producto adquiere suma atención, ya que se utiliza fundamentalmente como desinfectante de las vías respiratorias, expectorante y modificador de las secreciones bronquiales, en el tratamiento de la tuberculosis pulmonar. De igual manera, trasciende por su gran aplicación en la elaboración de perfumes, jabones, barnices, en la fabricación de cigarrillos mentolados y otros usos que se dan en la industria química y farmacéutica (Parada \& Romero, 1983).

Además, cabe la posibilidad en El Salvador, de defender la originalidad de sus famosas tortas de maíz, mejor conocidas como "Pupusas", el cual se perfila como un elemento identificador de la gastronomía salvadoreña reconocido al nivel internacional. Éste constituye para los miles de salvadoreños dispersos en muchos países del mundo, un producto nostálgico. 
TABLA 6

El Salvador Exportaciones hacia el mundo de productos potenciales a obtener Denominaciones Geográficas Protegidas (2010)

\begin{tabular}{|l|c|}
\hline \multicolumn{1}{|c|}{ PRODUCTOS } & MILES US\$ \\
\hline Café sin tostar y sin descafeinar & 170000000 \\
\hline Azúcar & 106000000 \\
\hline Medicamentos Preparados & 70000000 \\
\hline
\end{tabular}

Fuente: COMEX con base en cifras de PROCOMER, 2010.

En el caso de Nicaragua se han estimado esfuerzos limitados por buscar denominaciones para otros productos, en particular para el queso prensado de Santo Tomás, el cual se produce en la zona de Chontales. No obstante, puede perfilar otros productos con potencial para ostentar una denominación protegida, entre ellas sirve de ejemplo sus famosas "hamacas", reconocidas al nivel internacional y el ron Flor de Caña entre otros.

TABLA 7

Nicaragua Exportaciones hacia el mundo de productos potenciales a obtener Denominaciones Geográficas Protegidas (2009)

\begin{tabular}{|l|c|}
\hline \multicolumn{1}{|c|}{ PRODUCTOS } & MILES US\$ \\
\hline Café sin tostar y sin descafeinar & 191784481 \\
\hline Carne de especie bovina & 143623965 \\
\hline Queso & 53435209 \\
\hline Azúcar & 26445289 \\
\hline Ron & 11544296 \\
\hline Bananas o plátanos, frescos o secos & 8864408 \\
\hline
\end{tabular}

Fuente: Ministerio de Fomento, Industria, Comercio (MIFIC), 2010

\section{CONCLUSIONES}

En el marco de apertura comercial de las últimas décadas, las Denominaciones Geográficas Protegidas (DGP), se consideran instrumentos comerciales y signos de "valor agregado" que, aplicados a productos con grados de vinculación al territorio, sean consecuencia de factores humanos propios del origen de los productos o en los que se identifique conocimientos particulares y tradiciones de fabricación concretos, pueden proporcionar mayores posibilidades de venta en los mercados internacionales.

En razón, la implementación de esas herramientas comerciales se vierte como una excelente oportunidad comercial, al ayudar a productos endógenos y que representan símbolos de identidad regional, a mantener la competitividad, a apreciarlos como nichos diferenciales de originalidad y a obtener mejores prerrogativas de acceso a mercados, que deciden cada vez, mejorar su compra.

En particular, obtener un mejor acceso de los productos con distinciones geográficas al mercado de la Unión Europea, con el que se mantiene una estrecha y creciente relación comercial y con el que se supone incrementará, una vez que entre en vigencia el Acuerdo de Asociación. La idea se fortalece cuando discusiones internacionales en materia comercial, lo mismo que convenios y tratados bi y multinacionales, incluyen cada vez más, regulaciones del uso de los nombres geográficos, con el fin de evitar posteriores conflictos y obstáculos a la libre circulación de mercancías.

Así las cosas, el desarrollo y consolidación de un sistema de Denominación Geográfica se percibe para los productos centroamericanos sumamente viable, ya que la región goza de condiciones geográficas, edafoclimáticas, históricas y sociales así como de abundante patrimonio natural y cultural único e irrepetible, el cual se expresa en modos de elaboración propios en productos agrícolas, alimenticios y artesanales. 
Por consiguiente, no es extraño que surja en los últimos años, un creciente interés por proteger el prestigio de los nombres donde se originan los productos, ya que en caso contrario, algunos productores son conscientes de que verían perder cuota en el mercado y la presencia de sus productos sustituidos en la práctica, por otros distintos, pero con el mismo nombre.

Pese a ello, falta mucho por hacer, por ende, resulta necesario que las diferentes agrupaciones de productores organizadas y órganos de control nacional y regional, brinden la atención debida, para subsanar los factores limitantes que se presentan para establecer adecuadamente las distinciones geográficas. En particular que emprendan una serie de iniciativas para maximizar oportunidades de exportación, manteniendo el respeto por la biodiversidad, el rescate de los suelos, la preservación del ambiente y la protección del patrimonio común constituido por el prestigio de un nombre geográfico.

\section{REFERENCIAS}

Arias, R. \& Vives, I. (2005). Doka Estate: Historia y arquitectura de una hacienda cafetalera. Revista Patrimonio, 5(5), San José, Costa Rica.

Asociación Reguladora de las Normas de la Denominación de Origen de los Santos. (25 de julio de 2008). Entrevista. San Marcos de Tarrazú, Costa Rica.

Barquero, M. (2003, 24 de noviembre). Empresas "roban" las denominaciones geográficas: Pueblos procuran proteger sus marcas. La Nación. Recuperado el 6-julio-2008, de www.nación.com

Camacho, C. \& Gilles, R. (1992). Caracterización de la cadena del café en Guatemala. Recuperado el 2-julio-2008, de www.turismo20.com/profiles/blog/ show?id=932414:BlogPost:27990

Camacho, A. (2008, 3-9 de febrero). Queseros turrialbeños tramitan protección. El Financiero. Recuperado el 15-setiembre-2008 de: www.elfinancierocr.com

Camacho, A. (2008, 3-9 de marzo). Manza-té quiere proteger manzanilla nacional. El Financiero. Recuperado el 10-setimbre-2008 de: www.elfinancierocr.com
Castro, L. (2000, 9 de abril). Innovaciones en marcas. El Financiero. San José, Costa Rica. Recuperado el 15-setiembre-2008 de: www.elfinancierocr.com

Castillo, S. (2007). La hora del café gourmet en el istmo. El Financiero. San José, Costa Rica. Recuperado el 15-setiembre-2008, de www.elfinancierocr.com

Comunidad Autónoma de Galicia. (2005). Ley 2/2005, de 18 de febrero, de Promoción y Defensa de la Calidad Alimentaria Gallega. (93). Galicia, España. Recuperado el 15-agosto-2008 de: www.boe.es/g/es en el ámbito de potenciación de la calidad de la producción alimentaria

Convenio Centroamericano para la Protección de la Propiedad Industrial (1970). Ley No. 4543 del 18 de marzo de 1970.

Chacón, L. (2008, 24 y 6 de julio). Caficultor y miembro de la Asociación Reguladora en Normas de Origen de los Santos. Entrevista. Santa María de Dota, Costa Rica.

Correa, C. (1996). El acuerdo TRIPs y el comercio internacional: efectos sobre América Latina y el Caribe: instrumentación del acuerdo TRIPs. SELA (LADI/CEPAL/ SELA/III/RGPC/DT, 5.

Dardón, B. (2006, 24 de abril). Café guatemalteco marca el rumbo: catalogado por expertos internacionales como uno de los mejores cafés del mundo. Prensa Libre. Guatemala. Recuperado el 1-setiembre-2008 de: www.PrensaLibre.com

Echandi, R. (2008, 7 de julio). Estado de la negociación del Acuerdo de Asociación entre Centroamérica y la Unión Europea con vistas a la IV Ronda de Negociación. Conferencia. Universidad Estatal a Distancia. San José, Costa Rica.

Europeos saborean el café de Marcala. (2008, 21 de junio). La Tribuna. Tegucigalpa; Honduras. Recuperado el 20 de julio de 2008, de www.latribuna.hn/news/47/ARTICLE/36562/2008-06-21.html

García, M. (2001, 28 y 29 de noviembre). Algunos apuntes sobre la protección de denominaciones de origen en países de economías emergentes: La Comunidad Andina In: Simposio sobre la protección internacional de las indicaciones geográficas. Organizado por la Organización Mundial de la Propiedad Intelectual (OMPI) y la Dirección Nacional de la Propiedad Industrial (DNPI). Ministerio de Industria, Energía y Minería del Uruguay. Montevideo, Documento OMPI/GEO/MVD/01/4

González, B. (s.f) Informe de viabilidad de la implementación de una Denominación de Origen Protegida en la Montaña de Puca (Norte De Lempira - Sur de Santa Bárbara). Universidad Politécnica de Madrid. Recuperado 2-abril-2008 de: www.cafedehonduras.org/ihcafe/administrador/aa_archivos/documentos/inform_viabilidad.pdf.pdf 
González , G. (2008, 3-9 de marzo). Así es el consumidor europeo. El Financiero. Recuperado el 13-agosto-2008, de www.elfinancierocr.com

González, G. (2006, 2-8 de octubre). UE exigirá a país que distinga productos según regiones. El Financiero. San José, Costa Rica. Recuperado el 13-agosto-2008, de www.elfinancierocr.com

Granados, L. (2004). Indicaciones Geográficas y Denominaciones de Origen. Un aporte para su implementación en Costa Rica. IICA-PRODAR-MAG-CNP. Costa Rica.

Hall, C. (1991). El café y el dasarrollo histórico geográfico de Costa Rica. Editorial Costa Rica. San José, Costa Rica.

Hernández, L. (2000, 11 de noviembre). La importancia de la denominación de origen. Boletín de Propiedad Intelectual en México y el Mundo, (35). México. Recuperado el 20-julio-2008, de www.marcas.com.mx

Instituto del Café de Costa Rica (ICAFE). (2004, 10 de febrero). Asistencia Técnica en Denominaciones de Origen y Agroturismo Cafetalero: Productores, Beneficiadores, Comerciantes y Exportadores de Café. Circular N 1218. San José, Costa Rica.

López, M. (1996). Las Denominaciones de Origen. Cedecs Editorial. Barcelona, España.

Mata, R. (2008, 24 de junio). Caficultor, Ingeniero Agrónomo y miembro de la Asociación Reguladora en Normas de la Denominación de Origen de los Santos. Entrevista. San Lorenzo de Tarrazú, Costa Rica.

Ministerio de Comercio Exterior de Costa Rica (COMEX). (2006). Importancia para Costa Rica del proceso de negociación de un acuerdo de asociación con la Unión Europea. San José, Costa Rica.

Montero, A. (2007, 24 de febrero). Definir el origen mejora posición en el mercado. El Financiero. San José, Costa Rica. Recuperado el 20-julio-2008, de www.elfinancierocr.com

Morales, S. (2006, 7 a 13 agosto). Café orgánico va directo a Seattle. El Financiero. Recuperado el 20- julio-2008, de www.elfinancierocr.com

Organización Mundial de la Propiedad Intelectual (OMPI). (2007). Indicaciones geográficas: De Darjeeling a Doha. Revista de OMPI. Recuperado el 25-julio-2008 de www.wipo.int/portal/index.html.es
Organización Mundial de la Propiedad Intelectual (OMPI). (2001, 1 de enero). Protection of Geographical Indications Under the TRIPSO. Revista de OMPI Recuperado el 25-julio-2008, de www.wipo.int/portal/index.html.es

Pacheco, A. (2005). Ventajas para Costa Rica en la firma de un tratado de libre comercio con la Unión Europea. Antología de curso Estudios europeos e integración regional. Arnoldo Rubio Ríos (compilador). Universidad Nacional. Costa Rica.

Parada, A. \& Romero, J. (1983). Desarrollo de un proceso de producción de bálsamo de El Salvador utilizando una extracción con alcohol etílico. Universidad Centroamericana José Simeón Cañas. San Salvador, El Salvador.

Productores Agropecuarios Orgánicos de Juáres- Organicoop. (2007). Café Orgánico: Café Marcala. Sección de etiquetas. Honduras. Recuperado el 15-agosto-2008, de www.honduras.usembassy.gov/embajada/secciones/econ_coffee_sp.pdf ó www.organicoop.blogspot.com/2007/10/caf-de-centroamrica.html

Proyecto Alianza de Aprendizaje. (2008, 23 junio). Capacitan técnicos centroamericanos en denominaciones de origen de café. Recuperado el 25-julio-2008, de www. elnuevodiario.com

Rivera, M. (2008, 5 de julio). País lidera exportaciones a UE. Diario Extra. San José, Costa Rica.

Samper, M. (1991). El trabajo en la sociedad rural costarricense: 1840-1940. Colección Nuestra Historia. EUNEDUNA. San José, Costa Rica.

Sánchez, A. \&Villalobos, M. (2010). El análisis de las relaciones comerciales de Centroamérica y la Unión Europea desde una perspectiva de economía política. Madrid, España. Recuperado el 1-mayo-2011, de www.usc.es/congresos/xiirem/pdf/12.pdf

Secretaría de Integración Económica Centroamericana (SIECA). (2006). Relaciones comerciales y de cooperación entre Centroamérica y la Unión Europea. Guatemala.

Weil, J. (1995). Changing sources of livehood from the earth and sea in northwestern Costa Rica. Antropology of Work Review, 16(1-2)

\section{Recibido: 22 de noviembre de 2010 Aceptado: 30 de enero de 2011}

